\title{
International COVID-19 biomarkers colloquium
}

\author{
Richard C. Becker ${ }^{1}$. Diana A. Gorog $2,3,4$
}

Accepted: 30 October 2021 / Published online: 4 November 2021

(c) The Author(s), under exclusive licence to Springer Science+Business Media, LLC, part of Springer Nature 2021

The Food and Drug Administration defines a biomarker as a laboratory measure or a physical sign that is used in therapeutic trials as a substitute for a clinically meaningful endpoint that is a direct measure of how a patient feels, functions, or survives, and is expected to predict the effect of the therapy [1]. In 1998, the National Institutes of Health Biomarkers Definitions Working Group added a dimension to this definition, and included that a biomarker is: "a characteristic that is objectively measured and evaluated as an indicator of normal biologic processes, pathogenic processes, or pharmacologic responses to a therapeutic intervention". The World Health Organization offers a definition in the context of exposures, including environmental effects on human health and disease- a biomarker is a chemical, its metabolite, or the product of an interaction between a chemical and some target molecule or cell that is measured in the human body (www.who.org accessed 29 October 2021). Collectively, the ideal biomarker offers sensitivity, specificity, and is readily available at a low cost. As with most test platforms, biomarkers should provide a "value add" for specific patients and clinical scenarios to achieve optimal patient care (Fig. 1) [2].

The COVID-19 pandemic now totaling 243,857,028 cases and 4,953,246 deaths continues to teach the medical, scientific and lay communities about viral infectious disease in the modern era (covid19.who.int accessed October 27, 2021). Among the many lessons learned for the medical community is the potential for transmissibility and host

Richard C. Becker

BECKERRC@ucmail.uc.edu

1 University of Cincinnati College of Medicine, Cincinnati, $\mathrm{OH}, \mathrm{USA}$

2 National Heart and Lung Institute, Imperial College, London, UK

3 East and North Hertfordshire NHS Trust, Stevenage, Hertfordshire, UK

4 School of Life and Medical Sciences, Postgraduate Medical School, University of Hertfordshire, Hatfield, UK infectivity of the SARS-CoV-2 virus. Moreover, it quickly became evident that the virus could affect any organ, including the circulatory system, either directly via tissue tropism or indirectly from inflammatory and immune responses, leukocyte debris such as cell-free DNA and histones, cellular vesicles and RNA viral particles.

COVID-19 predisposes to small, intermediate and large vessel thrombosis of the venous, arterial and microvascular systems. The initial cases of COVID-19 and subsequent experience highlighted marked elevations in several circulating biomarkers, including but not limited to D-dimer, interleukin (IL)-6, C-reactive protein (CRP), fibrinogen, von Willebrand factor (VWF) and factor VIII. The question posed was whether these biomarkers offered important diagnostic, prognostic and management insights for patient care.

The primary objective of the International COVID-19 Biomarkers (ICODE) group was to assemble experienced clinicians, clinician-scientists and translational scientists for open discussions about COVID-19. The specific aim was to focus discussions on early biomarkers of coagulation, fibrinolysis, platelet biology, vascular injury and diagnostic platforms on which a foundation for optimal patient care would emerge.

The ICODE group included 10 scholars experienced, active and engaged in the study of COVID-19. Members represented diverse disciplines in medicine, including cardiology, vascular medicine, hematology, platelet biology, molecular biology and bioinformatics. The group met virtually each month to discuss emerging information and topics of clinical relevance.

The inaugural undertaking was to draft a series of focused reviews on biomarkers of thrombosis during the acute stage of COVID-19 with an emphasis on including the best available evidence, understanding that the field would evolve rapidly, and updates would be required in the future. Each review included in this issue of the Journal of Thrombosis and Thrombolysis represents the contribution of each member of the ICODE group working collaboratively during an unprecedented time in human history. 


\section{Optimal \\ Outcomes

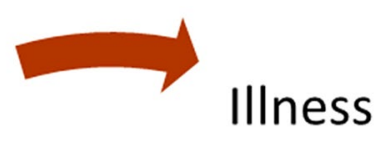

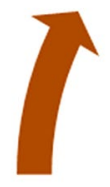

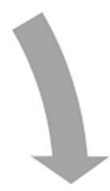

Prophylaxis

Treatment

\section{Biomarkers}

\section{References}

1. Ali M, Girgis S, Hassan A, Rudick S, Becker RC (2018) Inflammation and coronary artery disease: from pathophysiology to canakinumab anti-inflammatory thrombosis outcomes study (CANTOS). Coron Artery Dis 29:429-437

2. Becker RC (2007) Emerging paradigms, platforms, and unifying themes in biomarker science. J Am Coll Cardiol 50:1777-1780

Publisher's Note Springer Nature remains neutral with regard to jurisdictional claims in published maps and institutional affiliations.

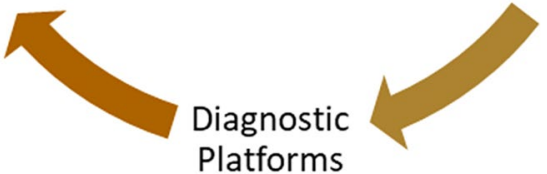

Fig. 1 A continuum of COVID-19 and the integration of biomarkers that inform diagnostic platforms, prophypaxis or treatment strategies, with a goal of optimal patient care 